\title{
Analyticity of Semigroups Generated by Singular Differential Matrix Operators
}

\author{
Ould Ahmed Mahmoud Sid Ahmed ${ }^{\mathbf{1}}$, Adel Saddi ${ }^{2}$ \\ ${ }^{1}$ Department of Mathematics, College of Science, Aljouf University, Aljouf, Saudi Arabia \\ ${ }^{2}$ Department of Mathematics, College of Education for Girls in Sarat Ebeidah, King Khalid University, Abha, Saudi \\ Arabia \\ E-mail: sidahmed@ju.edu.sa, adel.saddi@fsg.rnu.tn \\ Received March 9, 2010; August 3, 2010; August 6, 2010
}

\begin{abstract}
In this paper we prove the analyticity of the semigroups generated by some singular differential matrix operators of the form $\mathcal{A}=\frac{d^{2}}{d x^{2}}+\frac{B(x)}{x} \frac{d}{d x}$, in the Banach space $C\left([0,+\infty], M_{d}(C)\right)$, with suitable boundary conditions. To illustrate the work an example is discussed.
\end{abstract}

Keywords: Dissipative Operators, Positive Operators, Spectrum, Analytic Semigroups, Evolution Equations

\section{Introduction}

As we will see in the sequel the problem of characterizing operator matrices generating strongly continuous semigroups or analytic semigroups is quite difficult. The main problem consists in finding appropriate assumptions on the matrix entries allowing general results but still including the concrete examples we have in mind.

The evolution of a physical system in time is usually described in a Banach space by an initial value problem of the form

$$
\left\{\begin{array}{l}
\frac{d U(t)}{d t}-\mathcal{A} U(t)=0, \quad t \geq 0 \\
U(0)=U_{0} .
\end{array}\right.
$$

Problem of type (1.1) is well posed in a Banach space $X$ if and only if the operator $(\mathcal{A}, D(\mathcal{A}))$ generates a $C_{0}$-semigroup $\left(T_{t}\right)_{t>0}$ on $X$. Here the solution $U(t)$ is given by $U(t)=T_{t} U_{0}$ for the initial data $U_{0} \in D(\mathcal{A})$. For operator semigroups we refer to [1-5] and to [6] for the theory of operator matrices. The harmonic analysis for a class of differential operators with matrix coefficients was treated in $[7,8]$. In this work we are interested in a generalization of the analyticity and the positivity of the semigroup generated by a matrix singular differential operator $(\mathcal{A}, D(\mathcal{A}))$. A similar study was realized in [9] for a class of differential operators with matrix coefficients and interface. For the scalar case we refer to [10].
This paper is organized as follows. In the second section we introduce some notations and give preliminaries results. In the third section we investigate some properties of the operator $(\mathcal{A}, D(\mathcal{A}))$ in particular we prove that it is closed, densely defined, dissipative and satisfies the positive minimum principle. Some spectral properties of the operator $(\mathcal{A}, D(\mathcal{A}))$ are obtained in this section. In section fourth we established the analyticity of the semigroup generated by an operator in the form $(\mathcal{A}, D(\mathcal{A}))$. In this case the problem (1.1) has a unique solution for all $U_{0} \in X$. In section five we present a concrete example of application of the results obtained.

\section{Notations and Preliminary Results}

Let $M_{d}(C)$ be the space of $d \times d$ matrices with complex coefficients and $I_{d}$ the identity matrix. The norm of a matrix $A=\left(a_{i j}\right)_{1 \leq i, j \leq d} \in M_{d}(C)$ will be defined by

$$
\|A\|=\max _{1 \leq i, j \leq d}\left(\left|a_{i j}\right|\right)
$$

The reason of this special choice will be justified in Lemma 2.1 and Lemma 2.2.

A matrix $A=\left(a_{i j}\right)_{1 \leq i, j \leq d} \in M_{d}(C)$ is called nonnegative (resp positive) if all entries $a_{i j}$ are real numbers and nonnegative (resp positive). In this case we write $A \geq 0, \quad($ resp. $A>0)$ and we call $|A|=\left(\left|a_{i j}\right|\right)_{1 \leq i, j \leq d}$ 
the absolute value of $A$.

For $A=\left(a_{i j}\right)_{1 \leq i, j \leq d} \in M_{d}(C)$ and $A=\left(a_{i j}\right)_{1 \leq i, j \leq d} \in$ $M_{d}(C)$, we will write

$$
A \geq B \text { if } A-B \geq 0 .
$$

and

$$
A>B \text { if } A-B>0 .
$$

A matrix-valued functions is said to be continuous, differentiable or integrable if all its elements are continuous, differentiable or integrable functions. If the matrix $A(x)$ is integrable over $[a, b]$, then

$$
\left\|\int_{a}^{b} A(x) d x\right\| \leq \int_{a}^{b}\|A(x)\| d x .
$$

For two matrix functions $A(x)=\left(a_{i j}(x)\right)_{1 \leq i, j \leq d}$ and $B(x)=\left(b_{i j}(x)\right)_{1 \leq i, j \leq d}$, we shall write the negligibility $A(x)=o(B(x)), \quad x \rightarrow x_{0} \quad$ if, $a_{i j}(x)=o\left(b_{i j}(x)\right) x \rightarrow x_{0}$ for all $1 \leq i, j \leq d$. Similarly we define the notions of domination $A(x)=O(B(x))$ and the equivalence $A(x) \sim B(x) \quad$ (see for more details [11]).

Let $X$ be the space $C\left([0,+\infty], M_{d}(C)\right)$ of continuous, matrix-valued functions on $[0,+\infty]$. On the space $X$ we define the norm $\|\cdot\|_{X}$, by $\|f\|_{X}=\sup \|f(x)\|$, for all $f \in X$.

Note that the normed space $\left(X,\|\cdot\|_{X}\right)$ is a Banach space.

We denote by $\left.\left.C_{\infty}^{k}(] 0,+\infty\right], M_{d}(C)\right), k=1,2, \cdots$ the space of all $k$-times continuously differentiable matrixvalued functions $U$ defined on $] 0,+\infty[$ such that

$\lim _{x \rightarrow+\infty} U^{(p)}(x)$ exists and finite for all $0 \leq p \leq k$.

Consider a singular second order differential operator $(\mathcal{A}, D(\mathcal{A}))$ with matrix coefficients defined on $X$ by

$$
\mathcal{A} U=\frac{d^{2}}{d x^{2}} U+\frac{B(x)}{x} \frac{d}{d x} U,
$$

where $U$ and $B$ are matrix-valued functions, with the domain

$$
\begin{aligned}
& D(\mathcal{A})=\left\{U \in C\left([0,+\infty], M_{d}(C)\right) \cap \mathcal{C}_{\infty}^{2}(] 0,+\infty\right], \\
& \left.\left.M_{d}(C)\right), \lim _{x \rightarrow 0} \mathcal{A} U(x)=0\right\} .
\end{aligned}
$$

We assume that $B$ is a real valued continuous and bounded matrix-valued function on $[0,+\infty[$ and if $B(0)=-I_{d}$ we add the assumption

$$
R x^{D} \geq|B(x)-B(0)|
$$

in a neighborhood of 0 , for nonnegative constants diagonal matrices $R$ and $D$. Here $x^{D}$ is the diagonal matrix with diagonal entries $\alpha_{i}=x^{d_{i}}, i=1,2 \cdots, d$, where $d_{i}, i=1,2, \cdots, d$ are the diagonal entries of $D$.
We will now recall some results needed in the sequel.

Lemma 2.1 Let $A \in M_{d}(C)$. The following properties hold

1) $A \geq 0$ if and only if $A B \geq 0$ for all $B \geq 0$.

2) $|A B| \leq|A||B|$, hence $|A B| \leq|A||B|$ if $A \geq 0$.

Lemma 2.2 Let $A, B \in M_{d}(C)$. The following properties hold

1) $|A| \leq|B|$ implies $\|A\| \leq\|B\|$.

2) $\|A\|=\|A\|$.

3) $\|A E\| \leq d\|A\|$, where

$$
E=\left(e_{i j}\right)_{1 \leq i, j \leq d}, e_{i j}=1,1 \leq i, j \leq d .
$$

Proposition 2.1 Let $A$ be a nonnegative matrix with spectral radius $r(A)$.

1) The resolvent $R(\mu, A)$ is positive whenever $\mu>r(A)$.

2) If $|\mu|>r(A)$, then $|R(\mu, A)| \leq R(|\mu|, A)$.

Proof.

1) We use the Neumann series representation for the resolvent

$$
\begin{aligned}
& R(\mu, A)=(\mu-A)^{-1}=\mu^{-1}\left(I_{d}-\mu^{-1} A\right)^{-1} \\
& =\sum_{n \geq 0} \frac{A^{n}}{\mu^{n+1}} \quad \text { for }|\mu|>r(A) .
\end{aligned}
$$

If $A \geq 0$ then $A^{n} \geq 0$, for all $n$, hence for $\mu>r(A)$, we have

$$
R(\mu, A)=\lim _{n \rightarrow+\infty} \sum_{k=0}^{n} \frac{A^{k}}{\mu^{k+1}} \geq 0
$$

since the finite sums are positive and convergence holds in every entry.

2) For $|\mu|>r(A)$, we have

$$
\begin{aligned}
& |R(\mu, A)|=\left|\lim _{n \rightarrow+\infty} \sum_{k=0}^{n} \frac{A^{k}}{\mu^{k+1}}\right| \\
& \leq \lim _{n \rightarrow+\infty} \sum_{k=0}^{n} \frac{A^{k}}{|\mu|^{k+1}}=R(|\mu|, A) .
\end{aligned}
$$

Theorem 2.1 ([12], Perrons Theorem)

If $A$ is a nonnegative matrix, then $r(A)$ is an eigenvalue of $A$ with positive eigenvector.

Definition 2.1

1) An operator $(S, D(S))$ on a Banach Lattice $(X, \geq)$ is called positive if

$S u \geq 0$ for all $u \in D(S)_{+}=\{v \in D(S), v \geq 0\}$.

2) A semigroup $\left(T_{t}\right)_{t>0}$ on $X$ is positive if and only if $T_{t}$ is positive for all $t>0$.

Remark 2.1 An operator $(S, D(S))$ defined on a $\mathcal{C}(K)$-space (K compact) satisfies the positive minimum principle if for every $u \in D(S), u$ positive and $x \in K, u(x)=0$, then $(S u)(x) \geq 0$.

Theorem 2.2 ([1]) 
Let $(S, D(S))$ be the generator of a semigroup $\left(T_{t}\right)_{t>0}$ on $C(K)$, then the semigroup is positive if and only if $(S, D(S))$ satisfies the positive minimum principle.

\section{The Diagonal Case}

In this section we assume that the matrix function $B$ is diagonal.

\subsection{Characterization of the Operator $(\mathcal{A}, D(\mathcal{A}))$}

The proofs of Proposition 3.1 and Proposition 3.2 can be deduced from the scalar case given in Proposition 2.2 and Proposition 2.3 from [10].

Proposition 3.1 Let $-I_{d}>B(0)$ and $\left.\left.U \in X \cap C_{\infty}^{2}(] 0,+\infty\right], M_{d}(C)\right)$. Then $U \in D(\mathcal{A})$ if and only if $U \in \mathcal{C}^{2}\left([0, \tau], M_{d}(C)\right)$ for some $\tau>0$ and $U^{\prime}(0)=U^{\prime \prime}(0)=0$.

Proposition 3.2 Let $B(0)=-I_{d}$ and $\left.\left.U \in X \cap C_{\infty}^{2}(] 0,+\infty\right], M_{d}(C)\right)$. Then $U \in D(\mathcal{A})$ if and only if $\left.U \in C^{1}([0, \tau]), M_{d}(C)\right)$ for some $\tau>0$, $U^{\prime}(0)=0$ and

$$
\lim _{x \rightarrow 0} \frac{1}{x} \int_{0}^{x}\left(U^{\prime \prime}(x)-U^{\prime \prime}(t)\right) d t=0 .
$$

Moreover, if $U \in D(\mathcal{A})$ then $U^{\prime \prime}(x)=o(\log x E)$ and $\frac{1}{x} U^{\prime}(x)=o(\log x E)$ as $x \rightarrow 0$, where $E$ is the constant matrix introduced in Lemma 2.2.

Proposition 3.3 The operator $(\mathcal{A}, D(\mathcal{A}))$ is a densely defined, closed, dissipative and satisfies the positive minimum principle.

Proof. Put $B(x)=\left(\begin{array}{cccc}b_{1}(x) & 0 & \cdots & 0 \\ 0 & b_{2}(x) & \ddots & \vdots \\ \vdots & \ddots & \ddots & 0 \\ 0 & \cdots & 0 & b_{d}(x)\end{array}\right)$

and for $i=1,2 \cdots, d$, let $\mathcal{A}_{i} u=u^{\prime \prime}+\frac{b_{i}(x)}{x} u^{\prime}$

with

$$
\begin{aligned}
& D\left(\mathcal{A}_{i}\right)= \\
& \left.\left.\left\{u \in C([0,+\infty], C) \cap \mathcal{C}_{\infty}^{2}(] 0,+\infty\right], C\right), \lim _{x \rightarrow 0} \mathcal{A}_{i} u(x)=0\right\}
\end{aligned}
$$

Then $U=\left(u_{i j}\right)_{1 \leq i, j \leq d} \in D(\mathcal{A})$ if and only if $u_{i j} \in D\left(\mathcal{A}_{i}\right)$ for all $i, j=1,2 \cdots, d$. Hence from ([10]. Lemma 2.4), the operator $(\mathcal{A}, D(\mathcal{A}))$ is a densely defined and closed.

Let us show that $(\mathcal{A}, D(\mathcal{A}))$ is dissipative:
$\lambda\|U\|_{X} \leq\|\lambda U-\mathcal{A} U\|_{X}$, for $\lambda>0$ and $U \in D(\mathcal{A})$

Let $U=\left(u_{i j}\right)_{1 \leq i, j \leq d} \in D(\mathcal{A})$ and $\lambda>0$. According to [10], for all $1 \leq i, j \leq d$ we have

$$
\lambda\left\|u_{i j}\right\|_{\infty} \leq\left\|\left(\lambda u_{i j}-\mathcal{A}_{i} u_{i j}\right)\right\|_{\infty}
$$

then

$$
\lambda\|U\|_{X} \leq\|(\lambda U-\mathcal{A} U)\|_{X},
$$

and hence the dissipativity holds.

In order to prove that $(\mathcal{A}, D(\mathcal{A}))$ satisfies the positive minimum principle, assume that $U=\left(u_{i j}\right)_{1 \leq i, j \leq d} \in D(\mathcal{A})$ and positive such that $U\left(x_{0}\right)=0$ for some $x_{0} \in[0, \infty[$. If $x_{0}>0$ then $\left(u_{i j}\right) \geq 0$ and $u_{i j}\left(x_{0}\right)=0$, for all $i, j=1,2$, $\cdots, d$. Hence $u_{i j}{ }^{\prime}\left(x_{0}\right)=0$ and $u_{i j}{ }^{\prime}\left(x_{0}\right) \geq 0$ for all $i, j=$ $1,2, \cdots, d$. That means $\mathcal{A} U\left(x_{0}\right) \geq 0$. If $x_{0}=0$ then $\mathcal{A U}\left(\mathrm{x}_{0}\right)=0$.

\subsection{Spectral Analysis of the Operator $(\mathcal{A}, D(\mathcal{A}))$}

The purpose of next theorem is to deduce under reasonable hypothesis on the coefficients of $B$ a precise description of the spectrum of the operator $(\mathcal{A}, D(\mathcal{A}))$.

Theorem 3.1 If $-I_{d} \geq B(0)$, then the spectrum of $(\mathcal{A}, D(\mathcal{A}))$ is contained in $]-\infty, 0]$.

Proof. Let $\lambda \in C, U=\left(u_{i j}\right)_{1 \leq i, j \leq d} \in D(\mathcal{A})$ and $V=\left(v_{i j}\right)_{1 \leq i, j \leq d} \in X$.

We have $(\lambda-\mathcal{A}) U=V \Leftrightarrow\left(\lambda-\mathcal{A}_{i}\right) u_{i j}=v_{i j}$ for all $i, j=1,2, \cdots, d$ $\Leftrightarrow u_{i j}=\left(\lambda-\mathcal{A}_{i}\right)^{-1} v_{i j}$ for all $i, j=1,2, \cdots, d$ and $\lambda \in \bigcap_{i=1}^{i=d} \rho\left(\mathcal{A}_{i}\right)$.

This is sufficient to note that the spectrum of $(\mathcal{A}, D(\mathcal{A}))$ verifies $\sigma(\mathcal{A}) \subset \bigcup_{i=1}^{i=d} \sigma\left(\mathcal{A}_{i}\right)$ and then the result hold by ([10], Lemma 2.6).

Theorem 3.2 The operator $(\mathcal{A}, D(\mathcal{A}))$ with $-I_{d} \geq B(0)$, generates an analytic semigroup of angle $\frac{\pi}{2}$. Moreover, the semigroup is positive and contractive.

Proof. For $0<\theta<\pi$, put $\sum_{\theta}=\left\{z \in C^{*} ;|\arg (z)| \leq \pi-\theta\right\}$. It is clear from theorem 3.1 that $\sum_{\theta} \subset \rho(\mathcal{A})$ and then for $\lambda \in \sum_{\theta}$ and $i=1,2, \cdots, d$, the operator $\left(\lambda-\mathcal{A}_{i}\right)$ is invertible and verifies $\left\|\left(\lambda-\mathcal{A}_{i}\right)^{-1}\right\| \leq \frac{C_{i}}{|\lambda|_{i}}$ for some positive constant $C_{i}$ (see [10]). So $(\lambda-\mathcal{A})$ is invertible and veri- 
fies $\left\|(\lambda-\mathcal{A})^{-1}\right\|_{X} \leq \frac{\max _{1 \leq i \leq d} C_{i}}{|\lambda|}$. Hence the operator

$\left(\mathcal{A}, D(\mathcal{A})\right.$ ) with $-I_{d} \geq B(0)$, generates an analytic semigroup of angle $\frac{\pi}{2}$. For the positivity using the relation between the operators $\mathcal{A}_{i}, i=1,2, \cdots, d$ and $\mathcal{A}$ and from the fact that each operator $\left(\mathcal{A}_{i}, D\left(\mathcal{A}_{i}\right)\right)$, $i=1,2, \cdots, d$, generates an analytic positive and contractive semigroup, we deduce the result.

\section{The Non Diagonal Case}

In this section we consider the operator

$$
\mathcal{A} U=U^{\prime \prime}+\frac{B(x)}{x} U^{\prime}
$$

Assume that $B(x)=P(x) D(x) P^{-1}(x)$, where $\mathrm{D}$ is diagonal matrix function and $\mathrm{P}$ is nonsingular matrix function. If $P$ is constant, put $V=P^{-1} U$, then

$$
U=P V, U^{\prime}=P V^{\prime} \text { and } U^{\prime \prime}=P V^{\prime \prime}
$$

so we obtain

$$
P^{-1} \mathcal{A} P V=\left(V^{\prime \prime}+\frac{D(x)}{x} V^{\prime}\right)
$$

Hence, from Theorem 3.2 we can easily verify the following

Proposition 4.1 The operator $(\mathcal{A}, D(\mathcal{A}))$ with $-I_{d} \geq D(0)$, generates an analytic semigroup of angle $\frac{\pi}{2}$.

Remark that the condition $-I_{d} \geq D(0)$ is equivalent to the fact that the spectrum $\sigma(B(0))$ of the constant matrix $B(0)$ verifies $\sigma(B(0)) \subseteq]-\infty,-1]$.

We turn now to the general case in which we proceed with a perturbation argument. For this we recall the following definition.

Definition 4.1 ([2].Definition 2.1).

Let $A: D(A) \subset X \rightarrow X$ be a linear operator on the Banach space $X$. Any operator $B: D(B) \subset X \rightarrow X$ is called $A$-bounded if $D A) \subseteq D(B)$ and if there exist constants $a, b$ in $\mathbb{R}_{+}$such that

$$
\|B U\| \leq a\|A U\|+b\|U\| \text { for all } U \in D(A) .
$$

The $A$-bound of $B$ is $a_{0}=\inf \{a \geq 0$ : there exists $b \geq 0$ such that (5.1) holds $\}$

\section{Proposition 4.2 ([2].Theorem 2.10).}

Let the operator $(A, D(A))$ generates an analytic semigroup on a Banach space $X$. Then there exists a constant $\alpha>0$ such that $(A+B, D(A))$ generates an analytic semigroup for every $A$-bounded operator $B$ having $A$-bound $a_{0}<\alpha$.

Introduce now the operators $\left(\mathcal{A}_{0}, D\left(\mathcal{A}_{0}\right)\right)$ and $(\mathcal{R}, D(\mathcal{R})$ ) given by

$$
\mathcal{A}_{0}=\frac{d^{2}}{d x^{2}}+\frac{B(0)}{x} \frac{d}{d x} \text { and } \mathcal{R}=\frac{B(x)-B(0)}{x} \frac{d}{d x}
$$

with,

$$
\begin{aligned}
& D(\mathcal{R})=D\left(\mathcal{A}_{0}\right)=\{U \in C([0,+\infty], \\
& \left.\left.\left.\left.M_{d}(C)\right) \cap C_{\infty}^{2}(] 0,+\infty\right], M_{d}(C)\right), \lim _{x \rightarrow 0} \mathcal{A}_{0} U(x)=0\right\} .
\end{aligned}
$$

Then we have $\mathcal{A}=\mathcal{A}_{0}+\mathcal{R}$ and if we choose $D(\mathcal{A})=D\left(\mathcal{A}_{0}\right)$, we obtain the principal result of the paper.

Theorem 4.1 Assume that $B(0)$ is diagonalizable and $\sigma(B(0)) \subseteq]-\infty,-1]$ or $B(0)=-I_{d}$ and (2.2) holds. Then the operator $(\mathcal{A}, D(\mathcal{A}))$, generates an analytic semigroup of angle $\frac{\pi}{2}$.

Proof. Let $U \in D(\mathcal{A})$ and observe that

$$
\begin{aligned}
& \mathcal{A} U=\frac{d^{2}}{d x^{2}} U+\frac{B(x)}{x} \frac{d}{d x} U \\
& =\frac{d^{2}}{d x^{2}} U+\frac{B(0)}{x} \frac{d}{d x} U+\frac{B(x)-B(0)}{x} \frac{d}{d x} U \\
& =\mathcal{A}_{0} U+\mathcal{A} U,
\end{aligned}
$$

Let $\varepsilon>0$, there exists $\delta>0$ such that for all $0<x<\delta$ we have $\|B(x)-B(0)\|<\varepsilon$. The formula $\frac{U^{\prime}(x)}{x}=\int_{0}^{1} U^{\prime \prime}(x t) d t$ implies that

$$
\|R U(x)\| \leq \varepsilon\left\|U^{\prime \prime}\right\|_{X}, \quad 0<x<\delta .
$$

On the other hand from the Taylor expansion to order 2 at $x>\delta$ and for all $U \in D(\mathcal{A})$ there exists a constant $C_{\varepsilon}>0$ such that

$$
\begin{aligned}
& \|R U(x)\| \leq \varepsilon\left\|U^{\prime \prime}\right\|_{X}+C_{\varepsilon}\|U\|_{X} \\
& \leq \varepsilon\left\|\mathcal{A}_{0} U\right\|_{X}+\varepsilon\left\|\frac{B(0)}{x} U^{\prime}\right\|_{X}+C_{\varepsilon}\|U\|_{X} .
\end{aligned}
$$

Since $B(0)$ is diagonalizable and $\sigma(B(0)) \subset]-\infty,-1[$ or $B$ satisfies the condition (2.2) for $B(0)=-I_{d}$, the map

$$
U \mapsto \frac{B(0)}{x} \frac{d}{d x} U,
$$

from $D(\mathcal{A})$ into $C\left([0,+\infty], M_{d}(C)\right)$ is continuous (see [10]. Remark 2.5), so we deduce that the operator $\mathcal{R}$ is $\mathcal{A}$-bounded with $\mathcal{A}$-bound is equal to zero. Hence, the desired result follows by applying Theorem 3.2 and Proposition 4.2. 


\section{Application and Example}

Assume now that the operator $(\mathcal{A}, D(\mathcal{A}))$ satisfies the assumptions of Theorem 4.1, it generates so an analytic semigroup, and consider the evolution equation problem

$$
\left\{\begin{array}{l}
\frac{d U}{d t}(t)-\mathcal{A} U(t)=f(t), \quad t \geq 0 \\
U(0)=U_{0}
\end{array}\right.
$$

Corollary 5.1 If $f=0$ then the problem (5.2) has a unique solution for all $U_{0} \in X$. This solution is of infinitely continuously differentiable on ] $0,+\infty[$. [13].

For general case we have by Pazy [3] and R.autry

Corollary 5.2 If $f=\left(f_{i j}\right)_{1 \leq i, j \leq d}$, and for all $i, j, f_{i j} \in L^{1}(] 0, T[, C)$ and for every $\left.t \in\right] 0, T[$ there is a $\delta_{t}^{i, j}$ and a continuous function $\varphi_{t}^{i, j}:[0,+\infty[\rightarrow[0,+\infty[$ such that

$$
\left|f_{i j}(t)-f_{i j}(s)\right| \leq \phi_{t}^{i, j}(|t-s|) \text { and } \int_{0}^{\delta_{t}^{i, j}} \frac{\phi_{t}^{i j}(\tau) d \tau}{\tau}<\infty .
$$

Then the problem (5.2) has a classical solution.

\section{EXAMPLE}

Let the Banach space $X=C\left([0,+\infty], M_{d}(C)\right)$ and $\alpha \geq 0$. Put $\phi(x)=x^{\alpha}$ and define the linear transformation $P_{\phi}$ on $X$ in itself by $P_{\phi} U=U o \phi$. It is clear that $P_{\phi}$ is invertible and $\left(P_{\phi}\right)^{-1}=P^{-1}$.

Consider now the operator $(\mathcal{L}, D(\mathcal{L})$ ) defined on $X$ by

$$
\mathcal{L} U=x^{2-2 \alpha} U^{\prime \prime}+x^{1-2 \alpha} B(x) U^{\prime}
$$

with

$$
\begin{aligned}
& \left.D(\mathcal{L})=\left\{U \in X \cap C^{2}(] 0,+\infty\right], M_{d}(C)\right), \\
& \left.\mathcal{L} U \in X, \lim _{x \rightarrow 0}\left(\mathcal{L}_{0} P_{\phi}\right) U(x)=0\right\},
\end{aligned}
$$

and $B \in X$ is a diagonal matrix real valued function satisfying $B(0)<(1-2 \alpha) I_{d}$. A simple calculus gives

$$
\mathcal{L}=\alpha^{2} P_{\phi} \mathcal{K}\left(P_{\phi}\right)^{-1}
$$

where

$$
\mathcal{K} U=U^{\prime \prime}+\frac{1}{\alpha}\left(\frac{\alpha-1+B\left(x^{\frac{1}{\alpha}}\right)}{x}\right) U^{\prime} .
$$

Put

$$
\begin{aligned}
& D(\mathcal{K})=\left\{U \in C\left([0,+\infty], M_{d}(C)\right) \cap C_{\infty}^{2}(] 0,+\infty\right], \\
& \left.\left.M_{d}(C)\right), \lim _{x \rightarrow 0} \mathcal{K} U(x)=0\right\} .
\end{aligned}
$$

From Theorem 3.2 the operator $(\mathcal{K}, D(\mathcal{K}))$ generates an analytic semigroup of angle $\frac{\pi}{2}$ moreover, the semi- group is positive and contractive. Hence the relation (5.3) implies that the operator $(\mathcal{L}, D(\mathcal{L}))$ generates an analytic semigroup of angle $\frac{\pi}{2}$ and the semigroup is contractive if $\alpha \geq 1$.

\section{Acknowledgements}

The Authors wish to thank Professor A. Rhandi for many helpful discussions and comments on the manuscript.

\section{References}

[1] R. Nagel, "One-Parameter Semigroups of Positive Operators,” Lecture Notes in Math, Springer-Verlag, 1986.

[2] K. J. Engel and R. Nagel, "One-Parameter Semigroups for Linear Evolution Equations,” Springer-Verlag, 2000.

[3] A. Pazy, "Semigroups of Linear Operators and Applications to Partial Differential Equations," Applied Math Sciences 44, Springer, 1983.

[4] K. Ito and F. Kappel, "Evolution Equations and Approximations," Series on Advances in Mathematics for Applied Sciences, Vol. 61, 2002.

[5] E. M. Ouhabaz, "Analysis of Heat Equations on Domains,” Princeton University Press, New Jersey, 2005.

[6] K. J. Engel, "Operator Matrices and Systems of Evolution Equations.” (Preprint).

[7] N. H. Mahmoud, "Partial Differential Equations with Matricial Coefficients and Generalised Translation Operators," Transactions of the Americain Mathematical Society, Vol. 352, No. 8, 2000, pp. 3687-3706.

[8] N. H. Mahmoud, "Heat Equations Associated with Matrix Singular Differential Operators and Spectral Theory,” Integral Transforms and Special Functions, Vol. 15, No. 3, 2004, pp. 251-266.

[9] A. Saddi and O. A. M. S. Ahmed, “Analyticity of Semigroups Generated by a Class of Differential Operators with Matrix Coefficients and Interface," Semigroup Forum, Vol. 71, No. 1, 2005, pp. 1-17.

[10] G. Metafune, “Analyticity for Some Degenerate One-Dimentional Evolution Equations," Studia Mathematica, Vol. 127, No. 3, 1998, pp. 251-276.

[11] Z. S. Agranovich and V. A. Marchenko, "The Inverse Problem of Scattering Theory,” Kharkov State University, Gordon and Breach, NewYork and London, 1963.

[12] R. B. Bapat and T. E. S. Raghavan, "Nonegative Matrices and Applications," Cambridge University Press, Cambridge, 1997.

[13] R. Dautray and J.-L. Lions, "Analyse Mathématique et Calcul Numérique pour les Sciences et les Techniques,” Tome 3, Série Scientifique, Masson, 1985. 